\title{
Neuropsychological Outcome after Endoscopic Third Ventriculostomy
}

\author{
Walter J. Hader, Brian L. Brooks, Lisa Partlo, Mark Hamilton
}

\begin{abstract}
Background: Cognitive dysfunction is a common complaint associated with obstructive hydrocephalus. The purpose of this study was to determine the effect of endoscopic third ventriculostomy (ETV) on the neuropsychological outcome in patients presenting with cognitive decline and obstructive hydrocephalus. Methods: A retrospective review of patients who underwent ETV at the University of Calgary and had both pre and post operative neuropsychological testing, was completed. Presenting clinical features, etiology of hydrocephalus and ventricle size utilizing frontal occipital horn ratio was obtained. Outcomes and complications of the ETV were recorded. Detailed measures of intelligence, attention and concentration, executive function, visual and verbal memory, language functions and fine motor skills were completed. Post treatment change was determined utilizing Reliable Change Index. Results: A total of 13 patients were identified. Etiology of the hydrocephalus was aqueductal stenosis in 8 and tectal glioma in 4 . The majority of patients $(11$ of $13,85 \%)$ demonstrated cognitive dysfunction at the borderline $(\leq 1 \mathrm{SD})$ or impairment level $(\leq 1.5 \mathrm{SDs})$ in at least one domain. Nine patients $(69 \%)$ showed reliable improvement in at least one cognitive domain. The greatest improvement was seen with visual memory (42\%). One quarter to one third of patients demonstrated improvement on tests of intelligence quotient, verbal memory, attention and concentration, and executive function. Two patients declined in executive functioning. Ventricle size improved in eight patients. Conclusions: ETV is a safe effective procedure, capable of producing reliable improvements in cognitive dysfunction with hydrocephalus. Patients with cognitive complaints alone may benefit from ETV.
\end{abstract}

RÉSUMÉ: Issue neuropsychologique après ventriculostomie endoscopique du troisième ventricule. Contexte: La dysfonction cognitive est une complication fréquente de l'hydrocéphalie obstructive. Le but de cette étude était d'examiner les conséquences de la ventriculostomie endoscopique du troisième ventricule (VETV) sur l'issue neuropsychologique chez des patients présentant un déclin cognitif et une hydrocéphalie obstructive. Méthode: Nous avons effectué une revue rétrospective des dossiers de patients qui ont subi une VETV à l'Université de Calgary et chez qui des tests neuropsychologiques pré et postopératoires avaient été effectués. Les manifestations cliniques au moment de la consultation initiale, l'étiologie de l'hydrocéphalie et la taille du ventricule évaluée au moyen du ratio cornes frontale/occipitale ont été recueillies ainsi que l'issue et les complications de la VETV. De plus, des mesures détaillées de l'intelligence, de l'attention et de la concentration, de la fonction exécutive, de la mémoire visuelle et verbale, des fonctions langagières et de la motricité fine avaient été effectuées. Le changement après traitement a été évalué au moyen de l'Indice de changement fiable (Reliable Change Index). Résultats: Treize patients ont été identifiés. L'hydrocéphalie était due à une sténose de l'aqueduc de Sylvius chez 8 patients et à un gliome de la lame tectale du mésencéphale chez 4 patients. La majorité des patients, soit 11 patients sur 13 (85\%), présentaient une dysfonction cognitive limite (ÉT $\leq 1)$ ou un déficit (ÉT $\leq 1,5)$ dans au moins un domaine. Neuf patients (69\%) présentaient une nette amélioration dans au moins un domaine cognitif. Nous avons constaté le plus grand niveau d'amélioration dans le domaine de la mémoire visuelle (42\%). Entre le quart et le tiers des patients présentaient une amélioration des tests de quotient intellectuel, de mémoire verbale, d'attention et de concentration et de fonction exécutive. La fonction exécutive s'était détériorée chez deux patients alors que la taille du ventricule s'était améliorée chez 8 patients. Conclusions: La VETV est une intervention sûre et efficace, qui peut donner lieu à des améliorations notables de la dysfonction cognitive associée à l'hydrocéphalie. Les patients qui ne présentent que des troubles cognitifs peuvent tirer bénéfice de la VETV.

Keywords: Endoscopic third ventriculostomy, cognitive outcome, obstructive hydrocephalus

doi:10.1017/cjn.2014.108

Can J Neurol Sci. 2014; 41: 729-734

Cognitive dysfunction is an important clinical feature that may be identified at presentation of both acute and chronic untreated hydrocephalus, or at the time of shunt malfunction in previously treated patients. ${ }^{1}$ Detailed neuropsychological evaluation in patients with hydrocephalus has demonstrated that widespread cognitive difficulties may be present, including deficits in visual and tactile perception, attention ${ }^{2}$, memory ${ }^{3}$, visuospatial and motor function, and conceptualization and problem solving., The cognitive complaints may be associated with more typical symptoms of acute "high pressure" hydrocephalus, be part of a normal pressure hydrocephalus syndrome or may be the only complaint in a patient with obstructive hydrocephalus. When cognitive dysfunction is the sole presenting symptom and is associated with what appears to be chronic "arrested" hydrocephalus on neuroimaging, treatment is often deferred since clinicians believe that the patient's complaints, like the imaging, are not progressive and too severe in nature and are therefore unlikely to change with intervention.

Endoscopic third ventriculostomy (ETV) is a widely accepted treatment option for patients with obstructive hydrocephalus both

From the Department of Clinical Neurosciences (WJH, MH); Department of Psychology (LP), Department of Paediatrics (BLB), University of Calgary Alberta Children's Hospital Research Institute (BLB), Calgary, Alberta, Canada.

Received July 1, 2014. Final Revisions Submitted August 22, 2014

Correspondence to: Walter J. Hader, Department of Clinical Neurosciences, Alberta

Children's Hospital, 2888 Shaganappi Trail NW, T3B 6A8, Calgary, Alberta, Canada.

E-mail: walter.hader@albertahealthservices.ca 
as an initial treatment and at the time of shunt malfunction. ${ }^{6,7,8}$ Although the success of ETV for the majority of symptoms of obstructive hydrocephalus has been demonstrated and subjective improvement of cognitive symptoms has been reported, the objective cognitive benefits of intervention with ETV, particularly where the complaints are isolated, remains unclear. Reports of neurocognitive evaluation of patients evaluated only after ETV have revealed that deficits in memory and executive function are present. $^{9,10}$ The exact etiology of the cognitive inefficiencies, including whether unresolved dysfunction is associated with the hydrocephalus itself or related to previous shunting procedures, and/or surgical complications of the ETV itself is not known. Anatomical substrates important to cognitive functioning, in particular the frontal lobes and its white matter connections, and specific memory related structures including the fornix and mammillary bodies, are at risk during an ETV and any potential cognitive benefit of the ETV in treating the hydrocephalus may be lost with injury to these structures. It has been widely thought that ETV poses little risk to cognitive function, although subjective reports of short term memory difficulty exist. ${ }^{11}$ While injury to forniceal tracts resulting in severe amnesia has been reported with stereotactic $\mathrm{ETV}^{12}$, no reports detailing objective cognitive decline after ETV exist.

The purpose of this study is to determine the effect of ETV on the neuropsychological outcome in patients presenting with cognitive decline secondary to obstructive hydrocephalus.

\section{MeTHODS}

A retrospective review of patients identified from hydrocephalus databases at Foothills Medical Centre and Alberta Children's Hospital who underwent ETV for obstructive hydrocephalus and had both pre and post operative neuropsychological testing during the course of their treatment, was completed. All procedures were completed by two neuroendoscopic surgeons. The procedure has previously been described. ${ }^{7}$ Patient demographics, including age at surgery, presenting clinical features, duration of symptoms, etiology of obstructive hydrocephalus and measurement of the ventricle size utilizing the frontal occipital horn ratio $(\mathrm{FOR})^{13}$ on pre and post operative magnetic resonance imaging (minimum one year post ETV) was obtained. Outcome of the ETV including complications of the procedure, duration of follow up, and resolution of presenting symptoms was collected. A complete battery of neuropsychological tests were completed prior to and after surgery including measures of intelligence, attention and concentration, executive function, visual and verbal memory, language functions and fine motor skills. For the purpose of this study, the threshold for cognitive impairment on the various neuropsychological domains was considered at or below the $7^{\text {th }}$ percentile $(\leq 1.5$ standard deviation (SD) while borderline impairment was at or below the $16^{\text {th }}$ percentile $(\leq 1 \mathrm{SD})$.

Measures of post treatment cognitive change were determined utilizing the Reliable Change Index (RCI), which is based on an adaptation of Jacobson and Truax ${ }^{14}$ (1991) (see Figure 1 for the formula). The RCI determines whether a statistically reliable change has occurred when measurement error/test reliability and

RCI $(90 \%$ confidence interval $)= \pm 1.64 \times \sqrt{\left[\left(S D_{1} \sqrt{1-r_{12}}\right)^{2}+\left[\left(S D_{2} \sqrt{1-r_{12}}\right)^{2}\right]\right.}$

Figure 1: Reliable change index formula. practice effects are considered. A 90\% change score confidence interval can be derived based on this information, within which scores are expected to occur by chance. Differences that are of clinical significance (i.e., larger or greater than 1.5 standard deviations from the non-treated mean score), are thus clinically relevant gains or losses in the treatment group. In summary, by using the RCI we will assess whether changes in the surgery group are clinically meaningful compared to baseline-retest changes in age-matched controls for all patients. Measures of ventricular size change were compared utilizing Fischers exact T-Test (95\% confidence interval (CI)).

\section{Results}

\section{Patient Demographics}

A total of 13 patients ( 9 male: 4 female), all undergoing their first treatment for hydrocephalus, were identified. The mean age at surgery was $32(\mathrm{SD}=20.9)$ years. Six patients were under 20 at the time of surgery. The average age of the pediatric cohort was 13 years $(\mathrm{SD}=5.8)$, while the average age in the adult cohort was 48 years $(\mathrm{SD}=13.6)$. Etiology of the hydrocephalus was aqueductal stenosis in eight, tectal glioma in four and a posterior fossa cyst with fourth ventricle outlet obstruction in one. The primary complaint at presentation was headache in two patients and cognitive dysfunction in ten patients: four as part of an normal pressure hydrocephalus-like syndrome, and four with cognitive complaints alone. One adult patient presented with secondary amenhorrea and a child with blurred vision in addition to the cognitve dysfunction. A single pediatric patient had an enlarging head circumference. Twelve of thirteen patients had subjective and progressively worsening cognitive complaints prior to ETV upon systems review. Eleven of the patients had cognitive complaints of greater than one year prior to the ETV. Four patients had cognitive complaints for greater than five years, one for more than 25 years.

\section{ETV}

Twelve of thirteen patients underwent uncomplicated ETVs. One patient suffered a post operative chronic subdural hematoma, identified six weeks after the ETV procedure, requiring burrhole drainage followed by craniotomy for removal of the hematoma. Despite the complication, reliable gains in multiple cognitive domains were still seen in follow up for this patient. Post operative imaging completed revealed patent third ventriculostomies in all.

\section{FOR ratio}

Magnetic resonance imaging prior to ETV demonstrated evidence of acute hydrocephalus, with obvious transependymal flow, in three patients, while the remaining ten had chronic hydrocephalus. The mean FOR at diagnosis was $0.55(\mathrm{SD}=0.5)$ and $0.49(\mathrm{SD}=0.5)$ after ETV $(\mathrm{p}<0.05)$. Eight patients demonstrated improvement (1 SD) in ventricle size after ETV while five patients showed no change. All patients with acute hydrocephalus (three patients) and five of ten patients (50\%) with chronic hydrocephalus on preoperative imaging demonstrated objective improvement in ventricle size (see Figure 2). 


\section{Neuropsychological Examination}

The majority of patients (11 of $13,85 \%)$ demonstrated cognitive dysfunction at the borderline or impairment level in at least one domain prior to surgery. (See Table 1) Seven patients (54\%) had borderline or impaired function in at least two domains. No patient had an impairment of intelligence quotient (IQ), however a trend for lower IQ scores was present in younger patients (mean 37 percentile (\%ile) vs $64 \%$ ile). Impairments in verbal and visual memory were the most common deficits and present in $58 \%$ $(7 / 12)$ and $67 \%(8 / 12)$ of patients respectively. Attention and concentration deficits were identified in $33 \%$ (3/9), language deficits in $30 \%(3 / 10)$ and executive dysfunction was identified in $25 \%(3 / 12)$ of patients.
Postoperative cognitive testing was completed a mean of 15.8 (range $=6-42$ ) months after surgery. Assessment demonstrated stable findings or improvements on select neuropsychological tests in the majority of patients. (See Table 2). Nine of thirteen patients $(85 \%)$ showed reliable improvement in at least one cognitive domain that was evaluated in postoperative testing. The most frequent improvement in cognitive function was in visual memory (5/12) (42\%) (see Table 3). Overall, one-quarter to onethird of patients demonstrated reliable improvement on tests of IQ, attention and concentration, verbal memory and executive dysfunction. No reliable gains or losses in language function could be demonstrated in any patient. Three of six $(50 \%)$ pediatric age patients demonstrated reliable improvements in IQ whereas IQ was unchanged in all adult patients. The majority $(9 / 13(69 \%))$ of

\section{Table 1: Preoperative Neuropsychological Function}

\begin{tabular}{|c|c|c|c|c|c|c|c|}
\hline Patient & Age & Intelligence & Attention and Concentration & Verbal Memory & Visual Memory & Language & Executive \\
\hline 1 & 5 & 21 & 14 & 6 & 1 & - & 66 \\
\hline 2 & 9 & 27 & 27 & 7 & 4 & 73 & 61 \\
\hline 3 & 12 & 23 & 34 & 75 & 1 & 73 & 18 \\
\hline 4 & 17 & 37 & 13 & 91 & 25 & 97 & - \\
\hline 5 & 18 & 50 & 42 & 1 & 37 & 16 & 15 \\
\hline 6 & 20 & 63 & - & 69 & 69 & 12 & 63 \\
\hline 7 & 30 & 79 & 63 & 50 & 16 & 84 & 82 \\
\hline 8 & 35 & 42 & 21 & 16 & 1 & 5 & 5 \\
\hline 9 & 42 & 68 & 39 & 2 & 2 & 35 & 42 \\
\hline 10 & 49 & 86 & - & - & - & - & 87 \\
\hline 11 & 56 & 96 & - & 31 & 42 & 95 & 58 \\
\hline 12 & 62 & 32 & 1 & 2 & 1 & 38 & 3 \\
\hline 13 & 66 & 45 & - & 1 & 16 & - & 86 \\
\hline
\end{tabular}

All values in percentiles $($ mean $=50$, range $=1-99)$. Bold $=$ borderline $(\leq 16$ percentile $)$ or impaired $(\leq 7$ percentile $)$ performance.

$-=$ no data available.

Table 2: Reliable Change of Neuropsychological Function and Ventricle Size after ETV

\begin{tabular}{|c|c|c|c|c|c|c|c|c|}
\hline Patient & Age & Intelligence & Attention and Concentration & Verbal Memory & Visual Memory & Language & Executive & FOR \\
\hline 1 & 5 & $\mathrm{nc}$ & improve & $\mathrm{nc}$ & $\mathrm{nc}$ & - & $\mathrm{nc}$ & $\mathrm{nc}$ \\
\hline 2 & 9 & improve & $\mathrm{nc}$ & improve & $\mathrm{nc}$ & $\mathrm{nc}$ & worsen & improve \\
\hline 3 & 12 & improve & $\mathrm{nc}$ & $\mathrm{nc}$ & improve & $\mathrm{nc}$ & improve & improve \\
\hline 4 & 17 & $\mathrm{nc}$ & improve & $\mathrm{nc}$ & $\mathrm{nc}$ & $\mathrm{nc}$ & - & $\mathrm{nc}$ \\
\hline $5^{* *}$ & 18 & improve & $\mathrm{nc}$ & improve & improve & $\mathrm{nc}$ & improve & improve \\
\hline 6 & 20 & $\mathrm{nc}$ & - & $\mathrm{nc}$ & $\mathrm{nc}$ & - & worsen & nc \\
\hline 7 & 30 & $\mathrm{nc}$ & $\mathrm{nc}$ & $\mathrm{nc}$ & improve & $\mathrm{nc}$ & $\mathrm{nc}$ & improve \\
\hline 8 & 35 & nc & $\mathrm{nc}$ & improve & improve & $\mathrm{nc}$ & improve & improve \\
\hline 9 & 42 & $\mathrm{nc}$ & $\mathrm{nc}$ & $\mathrm{nc}$ & $\mathrm{nc}$ & $\mathrm{nc}$ & $\mathrm{nc}$ & $\mathrm{nc}$ \\
\hline 10 & 49 & $\mathrm{nc}$ & - & - & - & - & nc & improve \\
\hline 11 & 56 & nc & - & improve & improve & $\mathrm{nc}$ & $\mathrm{nc}$ & improve \\
\hline 12 & 62 & $\mathrm{nc}$ & improve & $\mathrm{nc}$ & $\mathrm{nc}$ & $\mathrm{nc}$ & improve & nc \\
\hline 13 & 66 & $\mathrm{nc}$ & - & $\mathrm{nc}$ & $\mathrm{nc}$ & - & $\mathrm{nc}$ & improve \\
\hline
\end{tabular}

Table note: For this study, 90\% confidence intervals were used for the RCI scores. See Figure 1 for the RCI calculation.

${ }^{* * *}$ patient with ETV complication. FOR: Frontal Occipital Horn Ratio; improve = > 1 SD change; nc = no change. 
Table 3: Summary of Reliable Change data for neuropsychological outcomes after ETV

\begin{tabular}{l|c|c|c|c}
\hline Neuropsychological Domain & & \multicolumn{3}{|c}{ Percent of Patients who... } \\
\hline & $\boldsymbol{n}$ & Worsen & Improve & No change \\
\hline Intelligence & 13 & 0.0 & 23.0 & 77.0 \\
\hline Attention and Concentration & 9 & 0 & 33.0 & 67.0 \\
\hline Verbal Memory & 12 & 0 & 33.0 & 75.0 \\
\hline Visual Memory & 12 & 0 & 42.0 & 58.0 \\
\hline Language & 9 & 0.0 & 0.0 & 100 \\
\hline Executive & 12 & 17.0 & 33.3 & 50.0 \\
\hline Subjective Assessment & 13 & 0 & 69.0 & 31.0 \\
\hline
\end{tabular}

Table note: For this study, $90 \%$ confidence intervals were used for the RCI scores. See Figure 1 for the RCI calculation.

patients reported subjective improvement in cognitive function after the ETV while four patients cited no change. Two of four patients who had no subjective improvement demonstrated a significant change on a single domain, one had improved visual memory and one executive dysfunction. Both objective and subjective cognitive improvements appeared to be more frequent in those patients who had reduction in ventricle size after ETV as demonstrated by a change in the FOR. Two pediatric patients showed a reliable worsening of executive functioning, both after uncomplicated ETVs. One of these patients demonstrated improvements in multiple other cognitive domains (IQ, verbal memory and motor hand speed bilaterally) while the other reported improved mood in the context of a stable cognitive profile.

\section{Discussion}

Demonstration of an objective reliable benefit of ETV in patients who present with symptoms of cognitive dysfunction has not been reported and few reports exist confirming that measures of cognitive function are unaffected after ETV. The results of our study show that successful ETV is capable of producing reliable objective improvements in cognitive dysfunction related to obstructive hydrocephalus. The majority of patients demonstrated benefits across a variety of cognitive domains including intelligence, attention and concentration, verbal and visual memory and executive function. While not feasible to measure progression of cognitive dysfunction in this population, the majority provided a history that indicated cognitive function was worsening. Patients with isolated cognitive dysfunction and a radiological picture of chronic "arrested" hydrocephalus may realize cognitive benefit from ETV, thus confirming that a chronic progressive "hydrocephalic encephalopathy", is reversible, even if only partially. This is consistent with a report of six patients with late onset idiopathic aqueductal stenosis who underwent ETV, most of whom had some impairments (1 SD or $2 \mathrm{SD}$ below mean) in executive and memory function. All of these patients showed improvements in their preoperative deficits. ${ }^{15}$ No reliable change methodology was utilized, however, to account for the degree of improvement that was identified. Larrson et $\mathrm{al}^{16}$ reported improvements in mental function, gait and incontinence after ETV in two patients with "compensated" hydrocephalus assessed with a detailed psychometric battery, although the extent of change similarly was not detailed. A report of a single patient presenting with headache and treated with ETV, detailed improvements with $90 \%$ confidence intervals in both recent and delayed memory and constructional ability utilizing The Repeatable Battery for the Assessment of Neuropsychological Status (RBANS). ${ }^{17}$ Ventriculo-peritoneal shunting has been previously demonstrated to provide cognitive improvements across multiple domains in children ${ }^{18}$ and adult patients ${ }^{16}$ with "asymptomatic" or compensated hydrocephalus and shunted and non shunted spina bifida patients with assumed arrested hydrocephalus. ${ }^{19}$ The ability to demonstrate similar cognitive benefits with ETV in patients with obstructive hydrocephalus may be attractive to neurosurgeons who might otherwise be reluctant to consider insertion of a ventriculoperitoneal shunt in patients with cognitive dysfunction and severe chronic hydrocephalus for fear of the potential associated complications of shunting this population.

Consistent with previous reports, hydrocephalus can affect a wide range of cognitive function in children and adults. A trend for the younger patients in this study to demonstrate IQ towards the lower end of the normal spectrum suggests an important additional impact of the obstructive hydrocephalus on the developing brain, which may be reversible for a certain time as demonstrated by half of pediatric patients realizing reliable improvements after ETV. Similarly, collateral negative neurocognitive consequences of the ETV may vary with respect to the age of the patient. Two patients, both pediatric in age, suffered reliable objective declines in executive function after otherwise uncomplicated ETVs. Although unproven, this may potentially be due to disruption of frontal white matter tracts with standard endoscopic approaches to the ventricular system. While no additional objective declines in other cognitive domains were identified in our study, Rekate ${ }^{11}$ reported new subjective short term memory deficits in a single patient after ETV for longstanding overt ventriculomegaly in adults. An organic personality disorder consisting of impulsiveness, aggression and impairment of memory was reported after ETV for slit ventricle syndrome. ${ }^{20}$ Damage to hypothalamic structures and forniceal systems at risk during the ETV approach to normal sized ventricles in a previously shunted patient was implicated in the pathogenesis. We have previously reported an increased complication rate of ETVs in those patients having an ETV at the time of shunt malfunction. ${ }^{7}$ Shunting, like ETV, may not be without its own cognitive risk, as new impairments in several cognitive domains have been reported after shunting spina bifida patients with chronic arrested hydrocephalus. ${ }^{19}$ Any possible neuropsychological risk of ETVs in children should be weighed against the risks of ventriculoperitoneal (VP) shunting and the risk to cognitive function with repeated shunt failure and revisions. ${ }^{21}$

Early studies which demonstrated the relationship of intelligence to cerebral cortical mantle thickness in infantile hydrocephalus $^{22}$ first raised the question about a relationship between ventricle size and neurocognitive function in hydrocephalus. Ventricular size reduction, although not essential for successful ETV, has been shown to be greater in patients with clinicallysuccessful ETV. ${ }^{23}$ Reduction in ventricular size after successful ETV, however, remains less than that seen with traditional VP shunting $^{24}$ and it has been suggested that ETV, while providing relief of high pressure symptoms, may not afford equivalent ventricular reduction and therefore as effective cognitive 
(A)

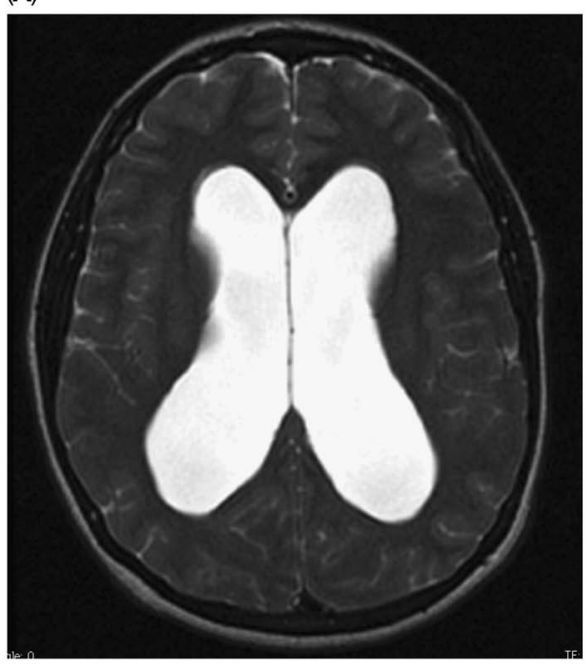

(B)

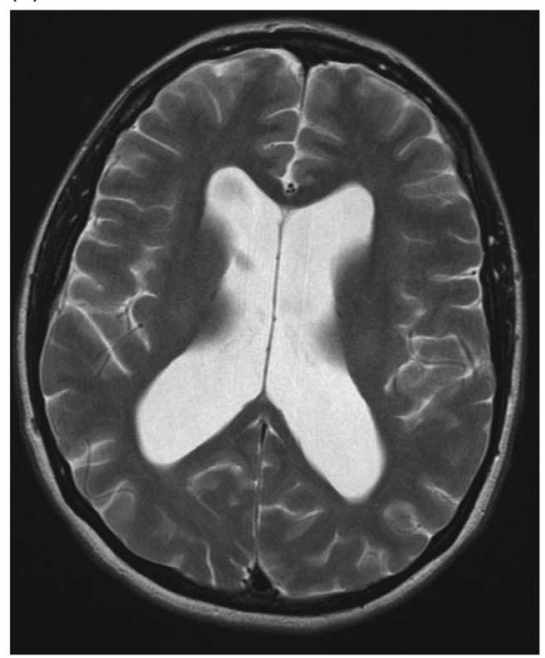

Figure 2: 35 year old female with global cognitive dysfunction with aqueductal stenosis. A. T2 weighted MRI demonstrates chronic hydrocephalus. B. T2 weighted MRI post ETV shows reduction in ventricle size and increase in subarachnoid spaces.

improvement. ${ }^{25}$ However, Warf et $\mathrm{al}^{26}$ reported that the neurocognitive outcome after ETV/choroid plexus cauterization was similar compared to VP shunting in a population of myelomeningocele patients and that ventricular volume, although larger than normal after the ETV, was no different between the groups. The majority of patients in our study who demonstrated significant improvements in ventricular size utilizing the FOR, appeared to realize greater improvements in neurocognitive functioning compared to those with stable ventricle size after the procedure. Diffusion Tensor Imaging improvements, which have been demonstrated in the absence of ventricular change after $\mathrm{ETV}^{27}$, may correlate better with neuropsychological improvement and serve as a more sensitive biomarker for assessing patients after treatment than ventricular size alone.

Limitations of the study performed include its retrospective nature. A variety of neuropsychological tests were completed in each domain, and in some cases, not all of the same tests were completed in the pre and post ETV assessments. Utilization of RCI was only completed if the same pre and post test was completed. Use of the RCI methodology may allow better comparisons between individuals even if different tests were used for the same domain. No quality of life measurements were completed in this study and therefore the impact of the changes noted on objective testing is uncertain, although the majority reported subjective improvements in cognitive function consistent with the results (i.e., none of the patients subjectively reported a decline in cognitive function). Additionally, our study did not address whether improvements in neurocognitive function identified correlated with the patients' ability to improve their day to day cognitive challenges, such as advancing their education or attaining meaningful employment. However, while we cannot ascertain with certainty the natural history of neurocognitive function in patients with chronic obstructive hydrocephalus, experience has shown that many of these patients worsen over time. Intervention with ETV may not only provide improvement but negate future declines. The exact cognitive threshold to support intervention in this patient group is yet to be determined.

\section{CONCLUSION}

ETV is a safe effective procedure capable of producing reliable objective improvements in cognitive dysfunction related to obstructive hydrocephalus. Declines in cognitive functioning following ETV are uncommon in pediatric and adult patients. Patients presenting with history of progressive cognitive dysfunction alone and with evidence of chronic obstructive hydrocephalus may also benefit from ETV.

\section{Disclosures}

Walter J. Hader does not have anything to disclose.

\section{REFERENCES}

1. Matson MA, Mahone M, Zabel A. Serial neuropsychological assessment and evidence of shunt malfunction in spina bifida: a longitudinal case study. Child Neuropsychol. 2005;11:315-32.

2. Mataro M, Junque C, Poca M, Sahuquillo J. Neuropsychological findings in congenital and aquired hydrocephalus. Neuropsycho$\log$ Rev. 2001;11(4):169-78.

3. Rourke BP. Syndrome of nonverbal learning disabilities: the final common pathway of white-matter disease/dysfunction? Clin Neuropsychol. 1987;1:209-330.

4. Dennis M, Fletcher JM, Rogers T, Hetherington R, Francis DJ. Objected and action-based visual perception in children with spina bifida and hydrocephalus. J Int Neuropsychol Soc. 2002;8:95-106.

5. Donder J, Rourke BP, Canady AI. Neuropsychological functioning of hydrocephalic children. J Clin Exp Neuropsychol. 1991;13:607-13.

6. Iantosca MR, Hader WJ, Drake JM. Results of endoscopic third ventriculostomy. Neurosurg Clin N Am. 2004;15:67-75.

7. Hader WJ, Walker R, Myles ST, Hamilton MG. Complications of endoscopic third ventriculostomy in previously shunted patients. Neurosurgery. 2008;63(1 suppl 1):168-76.

8. Drake JM. Endoscopic third ventricuolostomy in pediatric patients: the Canadian experience. Neurosurgery. 2007;60(5):881-6.

9. Al-Jumailly M, Jones B, Hayhurst C, et al. Long term neuropsychological outcome and management of "decompensated" longstanding overt ventriculomegaly in adults. Brit $\mathrm{J}$ Neurosurg. 2012;26(5):717-21. 
10. Lacy M, Oliveira M, Austria E, Frim MD. Neurocognitive outcome after endoscopic third ventriculostomy in patients with obstructive hydrocephalus. J Int Neuropsychol Soc. 2009; 15(3):394-8.

11. Rekate H. Longstanding overt ventriculomegaly in adults: pitfalls in treatment with endoscopic third ventriculostomy. Neurosurg Focus. 2007;22(4):E6.

12. Bonanni R, Carlesimo GA, Caltagirone C. Amnesia following endoscopic third ventriculostomy: a single case study. Eur Neurol. 2004;51:118-20.

13. Kulkarni AV, Drake JM, Armstrong DC, Dirks PB. Measurement of ventricular size: Reliability of the frontal and occipital horn ration compared to subjective assessment. Pediatr Neurosurg. 1999;31:65-70.

14. Jacobson NS, Truax P. Clinical significance: a statistical approach to defining meaningful change in psychotherapy research. J Consult Clin Psychol. 1991;59:12-9.

15. Burtscher J, Bartha L, Twerdy K, Eisner W, Benke T. Effect of endoscopic third ventriculostomy on neuropsychological outcome in late onset idiopathic aqeductal stenosis: a prospective study. J Neurol Neurosurg Psychiatry. 2003;74:222-5.

16. Larsson A, Stephensen H, Wikkelso C. Adult patients with "symptomatic" and "compensated" hydrocephalus benefit from surgery. Acta Neurolo Scand. 1999;99:81-90.

17. Hamada H, Hayashi N, Kurimoto M, Takaiwa A, Kurosaki K, Endo S. Neuropsychological changes after endoscopic third ventriculostomy for long-standing overt ventriculomegaly in adults. Neurol Med Chir (Tokyo). 2009;49:362-4.

18. Torkelson RD, Leibrock LG, Gustavson JL, Sundell RR. Neurological and neuropsychological effects of cerebral spinal fluid shunting in children with assumed arrested (normal pressure) hydrocephalus. J Neurol Neurosurg Psychiatry. 1985;48:799-806.
19. Mataro M, Poca M, Sahuquillo J, et al. Cognitive changes after cerebrospinal fluid shunting in young adults with spinal bifida and assumed arrested hydrocephalus. J Neurol Neurosurg Psychiatry. 2000;68:615-21.

20. Benabarre A, Ibanez J, Boget T, Obiois J, Martinez-Aran A, Vieta E. Neuropsychological and psychiatric complications in endoscopic third ventriculostomy: a clinical case report. J Neurol Neurosurg Psychiatry. 2001;71:268-71.

21. Ralph K, Moylan P, Canady A, Simmons S. The effects of multiple shunt revisions on neuropsychological functioning and memory. Neurol Res. 2000;22:131-6.

22. Young H, Nulsen F, Weiss M, Thomas P. The relationship of intelligence and cerebral mantle in treated infantile hydrocephalus (IQ potential in Hydrocephalic Children). Pediatrics. 1973; 52(1):38-44.

23. Kulkarni AV, Drake JM, Armstrong DC, Dirks PB. Imaging correlates of successful endoscopic third ventriculostomy. J Neurosurg. 2000;92:915-9.

24. Sainte-Rose C. Third Ventriculostomy in Manwaring KH, Crone K eds. Neuroendoscopy. New York: Mary Ann Liebert; 1992: 47-62.

25. Takahashi Y. Long-term outcome and neurologic development after endoscopic third ventriculostomy versus shunting during infancy. Childs Nerv Syst. 2006;22:1591-602.

26. Warf B, Ondoma S, Kulkarni A, et al. Neurocognitive outcome and ventricular volume in children with myelomeningocele treated for hydrocephalus in Uganda. J Neurosurg Pediatr. 2009;4(6): 564-570.

27. Buckley R, Weihong Y, Mangano F, et al. Longitudinal comparison of diffusion tensor imaging parameters and neuropsychological measures following endoscopic third ventriculostomy for hydrocephalus. J Neurosurg Pediatr. 2012;9:63-5. 